\section{From the Desk of Editor in Chief}

Editorial on: "Internet Addiction: The Emergence of a New Clinical Disorder" highlighted the effect of excessive interest to internet use leading to addiction. This leads to academic failure, marital discord and sometimes separation. Steps should be taken to limit the use of internet to reduce the menace of addiction and encourage physical activates like sports other cultural activities alternately .

Original Article on "Knowledge and Practice of Drinking Safe Water Among the Community People of Horintana, Khulna" explored the knowledge and practice of drinking safe water among the community people. Majority of the respondents $(84.5 \%)$ know about safe sources of drinking water but its pity that a large proportion (14.09\%) does not know about waterborne diseases. Proper health education is needed for this.

Article on "Estimation of Serum Copper in Postmenopausal Women Attending in a Tertiary Care Hospital, Bangladesh" found copper is essential for proper functioning of organs and metabolic process. Copper imbalance leads to impaired immune function, increase risk of cardiovascular and neurodegenerative disease. This should be borne in mind of clinicians.

Article on "Effects of Controlling of Blood Glucose in Adult Non-Diabetic Patients Undergoing Mitral Valve Replacement" shouwed blood glucose value of $10.1 \mathrm{mmol}$ or more after MVR under CPB is important issue related to postoperative morbidity and mortality. So proper glycamic control is necessary for MVR patients.

Article on "Declining Prevalence of Helicobacter Pylori Infection- A 13C Urea Breath Test (UBT) Based Study in Symptomatic Subjects in Dhaka, Bangladesh" showed that improving sanitary and hygienic condition of this region along with significantly increasing HP eradication therapy and PPI use by the symptomatic individuals contributed to the decreasing trend of infection.

Article on "A Study of Thyroid Profile in Patients with Benign Breast Disease" showed that thyroid profile is important useful investigation in associated benign breast disease. About $14.9 \%$ patients showed associated hypothyroidism and among this hypothyroid patients $4 \%$ showed hyperprolacteneima. So benign breast disease patient should be advised for thyroid profile. All of these patients were normal with proper treatment

Article on "Insulin secretory defects and determinants of attending at a tertiary hospital in northern region of Bangladesh" concluded that Both (HOMA\%B) and (IR) constitute the basic defects of diabetes but (HOMA\%B) seems to be more predominant in these subjects. (HOMA\%B) is associated with males and level of education and insulin resistance (IR) is associated with males and daily $\mathrm{CHO}$ intake.

Article on "Maternal Socioeconomic Factors Affecting Birth Weight of New Born Babies Born in Combined Military Hospital Dhaka" suggest that a substantial evidence of socioeconomic factors like family income, maternal education and social status has an effect on birth weight. Utilizing health care facilities, better training, educating mother, addressing demographic factors, condition can be improved.

Article on "Comparative Study of Dexmedetomidine and Fentanyl as an Adjuvant to Epidural Bupivacaine for Post-operative Pain Relief in Hepato-biliary Pancreatic Surgery" showed that dexmedetomidine is an ideal adjuvant to epidural bupivacaine for post operative analgenia compared to fentanyl in patients undergoing hepatobilliary \& pancreatic surgery.

Article on "Pattern of Admission and Outcome in a Neonatal Intensive Care Unit (NICU) of a Tertiary Care Hospital in Dhaka, Bangladesh" showed that prematurity, infection and birth asphyxia are the major causes of neonatal morbidity and mortality in hospital admitted neonats. These three should be borne in mind of paediatrician.

Article on "Role of Intravenous Paracetamol as PreEmptive Analgesic for Laparoscopic Cholecystectomy" indicated that pre emptive administration of IV paracemtamol in patients 
undergoing laproscopic surgery ensures an effective postoperative analgesic and reduces opioid consumption.

Article on "Use of Dexmedetomidine for Preventing Pain on Propofol Injection: A Double Blinded Placebo Controlled Study" reveaed efficacy of Dexmedetomidin for the prevention of pain cause by Propofol Injection used for general anesthesia .

Review Article on "Chromobacterium violaceum, a Rare but Fatal Bacteria Needs Special Clinical Attention" highlighted the importance of this saprophytic bacillus of soil and water which can be a cause of abscess in different sites, pneumonia, osteonmyelitis, shock and multidrug resistance and relapses.

Case Report on "Sinus Histocytosis with Splenomegaly in Children- A Rare Case Report" reveals sinus histocytosis been rare proliferative disease wich usually present with lympadenopathy but can present as spenomaglally so any case of speenmegally should be screened for sinus Histocytosis.
Case report on "Vasculitis Retinae- A Rare Presentation of SLE" reported about a rare presentation of SLE a rare complex auto immune disease as Vasculitis Retinae.

Case report on Ovarian Pregnancy- "A Rare Case Reported a rare ovarian pregnancy" with the complain of hypogastric pain and menorrhagia histopathology examination confirmed ovarian prgeancy. Although ovarian pregnancy is rare however any pregnant patient presented with hypogastric pain and menorrhagia should be screened for ectopic pregnancy.

Overall this issue will be very attractive to the reader like all other previous issues and will add some new knowledge \& create research interest in interested topic.

Thanks

Prof. Dr. Md. Tahminur Rahman

MBBS, M. Phil, PhD

Editor in Chief, AKMMC Journal 